\title{
Pathogen profile and risk factors of aerobic vaginitis in pregnant women: a retrospective cohort study
}

\author{
Na Li, Yi Yue, Qi Chen \\ Clinical Laboratory, the Affiliated Wuxi Maternity and Child Health Care Hospital of Nanjing Medical University, Wuxi, China \\ Contributions: (I) Conception and design: N Li, Y Yue, Q Chen; (II) Administrative support: Q Chen; (III) Provision of study materials or patients: N \\ Li, Y Yue; (IV) Collection and assembly of data: N Li; (V) Data analysis and interpretation: N Li; (VI) Manuscript writing: All authors; (VII) Final \\ approval of manuscript: All authors. \\ Correspondence to: Qi Chen. Clinical Laboratory, the Affiliated Wuxi Maternity and Child Health Care Hospital of Nanjing Medical University, No. \\ 48 Huaishu Lane, Wuxi 214000, China. Email: chenqi401871@163.com.
}

Background Vaginitis is one of the most common gynecological diseases in women and may severely affect the quality of life in patients. However, very few studies have investigated aerobic vaginitis (AV) in pregnant women, and our study was designed to identify the pathogen profile, clinical importance, and risk factors of $\mathrm{AV}$ in pregnancy.

Methods: This was a retrospective cohort study enrolling 685 women who attended our hospital between July 2018 and June 2020. Based on the incidence of AV, enrolled women were divided into an AV group and healthy control group, and demographic and clinical characteristics were retrospectively collected by two independent investigators. Some pathogens of AV were tested using quantitative real-time polymerase chain reaction for higher accuracy. Pregnancy outcomes were also retrospectively collected. Univariable and multivariable logistic regression analysis was used to determine the risk factors of AV incidence and adverse pregnant outcomes.

Results: Enrolled women were divided into an AV group of 182 women and healthy control group of 503 women. The proportions of women with a history of cesarean delivery and history of vaginal infection differed between the groups $(\mathrm{P}=0.002$ and $<0.001$, respectively). The mean gestational week at diagnosis of $\mathrm{AV}$ was $22.3 \pm 8.6$ weeks, and the most common pathogen of AV was Escherichia coli (28.6\%). After adjustment using multivariable logistic regression, a history of vaginal infection acted as an important risk factor of AV incidence, while a history of cesarean delivery, college education or above, and being employed could protect pregnant women from AV. In addition, the incidences of preterm birth, premature rupture of membranes, neonatal jaundice, and neonatal infection were much higher in the AV group than in the control group, showing significant difference $(\mathrm{P}<0.001,<0.001,=0.007$, and $=0.025)$. After adjustment using multivariable logistic regression, the incidence of $\mathrm{AV}$ and older age were important risk factors of premature rupture of membranes and neonatal infection.

Conclusions: Compared with healthy pregnant women, the presence of AV may increase the incidence of adverse outcomes. More attention should be paid to pregnant women with a history of vaginal infection.

Keywords! Pathogen profile; risk factors; aerobic vaginitis (AV); pregnant women; retrospective cohort study

Submitted Jun 02, 2021. Accepted for publication Jul 23, 2021.

doi: 10.21037/apm-21-1710

View this article at: https://dx.doi.org/10.21037/apm-21-1710 


\section{Introduction}

Vaginitis is a common gynecological disease in women of all ages (1), the prevalance of which varies with living habits, living environment, religious beliefs, and economic status. Several pathogens may cause the disease, and may take the form of bacterial vaginitis, mixed vaginitis, Candida vaginitis, Mycoplasma vaginitis, and Trichomonas vaginitis (2), the latter having been the most common type in past decades (3). However, the incidence of Trichomonas vaginitis has been decreasing in recent years, while that of bacterial vaginitis has remained stable (4). Aerobic vaginitis (AV) is more common than anaerobic vaginitis among all bacterial vaginitis (5), and the pathogens of $\mathrm{AV}$ include aerobic microorganisms from the gastrointestinal tract such as Escherichia coli, staphylococcus aureus, coagulase-negative staphylococci and group B streptococcus $(6,7)$. Patients with AV may suffer from abnormal vaginal discharge and vulvae itching and discomfort, which severely affect their quality of life.

During pregnancy, women will experience various hormonal and physiological changes, which also include changes in the vaginal environment. This may lead to an increased incidence rate of vaginitis and changes to pathogens (8). It has been reported that the pathogen profile of $\mathrm{AV}$ in late pregnancy differed from that in nonpregnant women in a Chinese population, with more group B streptococcus and less coagulase-negative staphylococci and staphylococcus aureus (9). The occurrence of AV may worsen the outcomes of pregnant women, resulting in more spontaneous preterm delivery and premature rupture of membranes (9-11). However, previous studies mainly focused on early or late stage pregnancy, and it is hard to understand the incidence of vaginitis across the whole of pregnancy. The study of risk factors of vaginitis in pregnant women also has important clinical significance. Many studies have explored the risk factors of vaginitis in nonpregnant women (12-14), which may differ significantly from those of non-pregnant women, due to hormonal and physiological changes. However, few studies have explored this issue to date, and the risk factors of $\mathrm{AV}$ in pregnant women remain unclear.

This study was designed to determine the prevalence and pathogen profile of $\mathrm{AV}$ in women at all stages of pregnancy and investigate the effects of AV on pregnancy outcomes. Most importantly, this study aimed to explore the risk factors of $\mathrm{AV}$ in pregnant women. The results may assist pregnant women reduce the incidence of $\mathrm{AV}$ and improve pregnancy outcomes. We present the following article in accordance with the STROBE reporting checklist (available at https://dx.doi.org/10.21037/apm-21-1710).

\section{Methods}

\section{Participant enrollment}

We retrospectively enrolled pregnant women who were admitted to the Wuxi Maternity and Child Health Care Hospital of Nanjing Medical University between July 2018 and June 2020, and divided them into a group with $\mathrm{AV}$ and a healthy control group. Women older than 18 years old and those who constantly received prenatal examination in our hospital were included, while those who were diagnosed with vaginitis other than $\mathrm{AV}$ or mixed vaginitis, did not attend for regular prenatal examinations and were lost to follow up, who received long term antibiotic treatment, who needed to terminate their pregnancy because of severe complications, were excluded. This study was approved by the Ethics Committee of the Affiliated Wuxi Maternity and Child Health Care Hospital of Nanjing Medical University (No. 20200037) and was conducted in compliance with the ethical principles of the Declaration of Helsinki (as revised in 2013). Written informed consent was not required due to the retrospective nature of the study.

\section{Data collection}

Following enrolment, the baseline characteristics of patients was collected including age, body mass index, multifetal pregnancy, parity, history of cesarean delivery, history of vaginal infection, history of hypertension and diabetes mellitus, level of education, history of smoking, and occupational status. The data were collected by two independent investigators to minimize errors.

All enrolled participants underwent a gynecological examination, and a sterile cotton swab was used to obtain samples of vaginal discharge. The diagnosis of $\mathrm{AV}$ was determined by lactobacillary grade, leukocyte number, proportion of toxic leukocytes, background flora, and proportion of parabasal epitheliocytes according to microscopic examination. Pathogens of $\mathrm{AV}$ were tested by conventional bacterial culture and biochemical analysis. Part pathogens were tested using quantitative real-time polymerase chain reaction for amplification of specific targets, followed by specific probe test for bacterial vaginosis markers, including Enterococcus group, bacterial vaginosis-associated bacterium 2, and Megasphaera 1 . The 


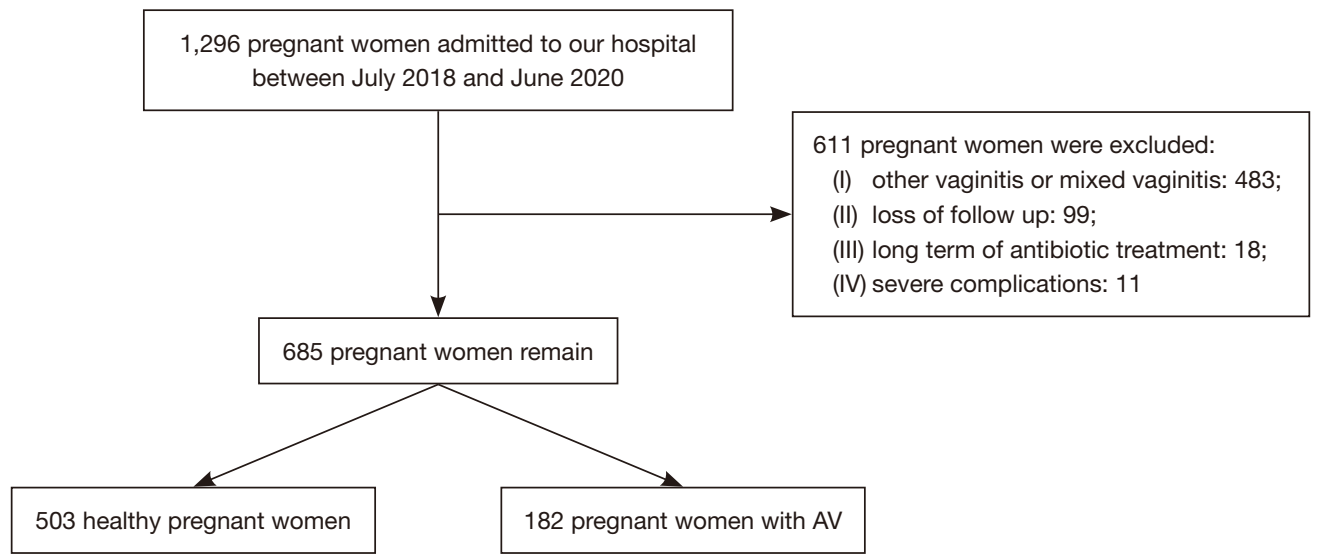

Figure 1 Flow chart of this study. AV, aerobic vaginitis.

gestational week of participants when diagnosed with AV was also recorded.

\section{Pregnancy outcomes}

All participants were followed up until 1 month after delivery. Some pregnant outcomes were recorded in this study for further analysis, including delivery mode, preterm birth, premature rupture of membranes, birth weight, Apgar score, neonatal jaundice, neonatal infection, and stillbirth.

\section{Statistical analyses}

Continuous variables were shown as mean with standard deviation and categorical variables were shown as number with percentage. Comparisons between continuous variables were analyzed using student $t$-test and those between categorical variables using Chi-square test. Univariable logistic regression analysis was performed to preliminarily determine the risk factors of adverse pregnant outcomes and AV. Multivariable logistic regression analysis was then used to verify the real risk factors after adjusting confounding variables according to univariable logistic regression analysis. Statistical analysis was performed using SPSS 20.0 (IBM Corporation, NY, USA) and P value less than 0.05 was considered statistically significant.

\section{Results}

We enrolled pregnant women who had attended our hospital over a 2 -year period and the flow chart is shown in Figure 1. In total, 1,296 women were admitted and 685 were enrolled in the study after 611 women failed to meet the exclusion criteria. Those included in the study were then divided into an $\mathrm{AV}$ group of 182 women and a healthy control group of 503 .

Demographic and clinical characteristics of both groups are shown in Table 1. In the control group, the mean age was $27.7 \pm 6.0$ years and mean body mass index was $24.0 \pm 4.9$. Four women had multifetal pregnancies, and more than half of the control group were primiparas. A history of cesarean delivery was seen in 101 women, 48 women had a history of vaginal infection, 40 had a history of smoking, 15 had hypertension, and eight women had diabetes. High school education or below was recorded in 322 women in the control group, and 419 were currently employed.

In the $\mathrm{AV}$ group, the mean age was $26.8 \pm 5.4$ years and mean body mass index was $23.9 \pm 11.0$. Three women had multifetal pregnancies and 117 women were primiparas. A history of cesarean delivery was seen in 18 women, 41 women had a history of vaginal infection, 11 had a history of smoking before pregnancy, four had hypertension, and two had diabetes mellitus. Around $75 \%$ of women in the $\mathrm{AV}$ group had received high school education or below, and 139 women were employed.

Comparison of the demographic and clinical characteristics in the AV group and control group showed significantly more women in the control group had a history of cesarean delivery ( $20.1 \%$ vs. $9.9 \%, \mathrm{P}=0.002)$, while $22.5 \%$ of the AV group had a history of vaginal infection, which was much more than the $9.5 \%$ in the control group $(\mathrm{P}<0.001)$. More women in the $\mathrm{AV}$ group received high school education or below compared to the control group $(75.8 \%$ vs. $64.0 \%, \mathrm{P}=0.004)$, and the proportion of 
Table 1 Demographic and clinical characteristics

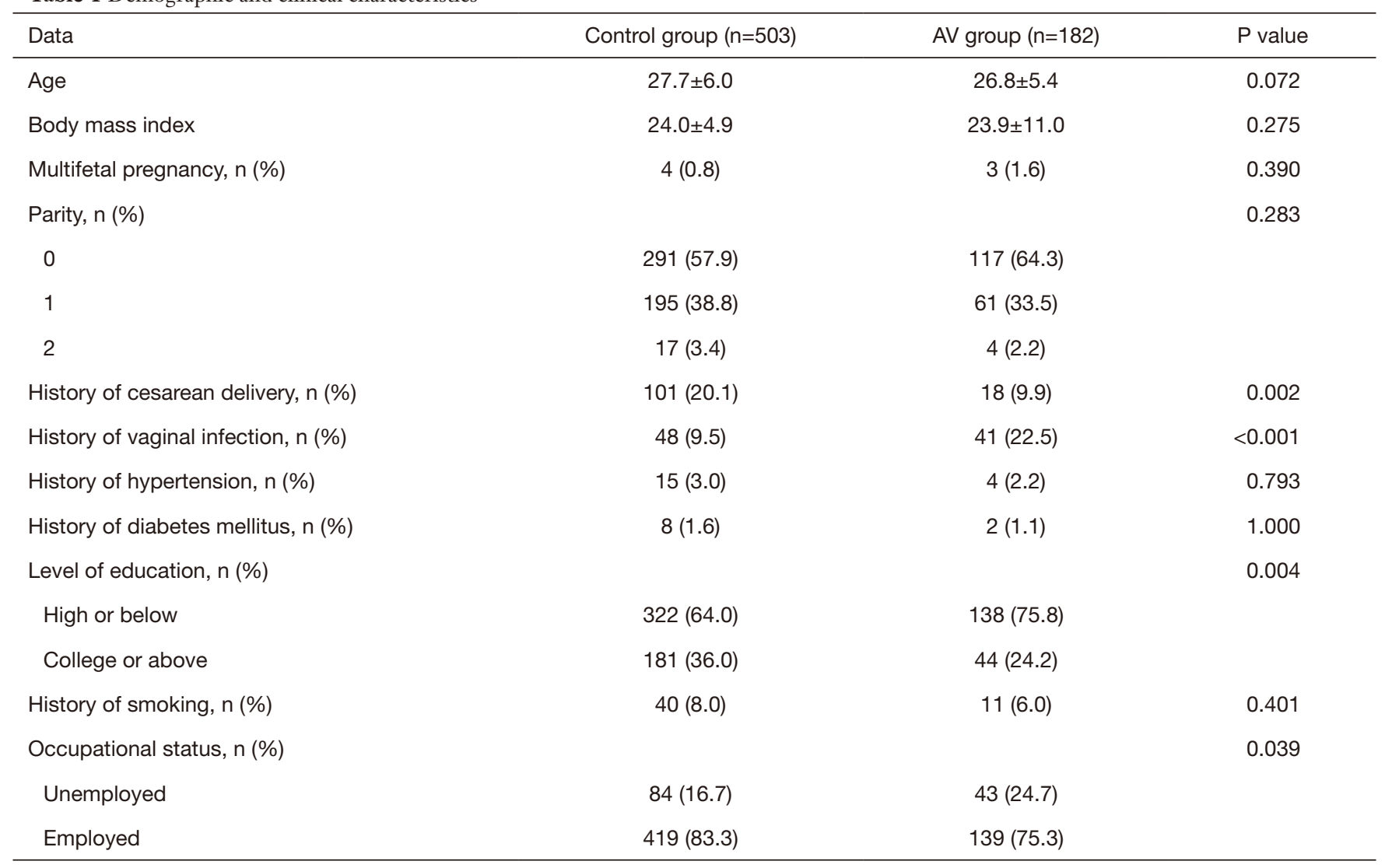

$\mathrm{AV}$, aerobic vaginitis.

Table 2 Distribution of bacterial pathogens in pregnant women with AV

\begin{tabular}{lc}
\hline Data & Number $(\mathrm{n}=182)$ \\
\hline Gestational week at diagnosis & $22.3 \pm 8.6$ \\
Pathogens, $\mathrm{n}(\%)$ & \\
Escherichia coli & $52(28.6)$ \\
Group B streptococcus & $44(24.2)$ \\
Enterococcus faecalis & $32(17.6)$ \\
Enterococcus faecium & $15(8.2)$ \\
Coagulase-negative staphylococci & $13(7.1)$ \\
Staphylococcus aureus & $11(6.0)$ \\
Lactobacillus & $9(4.9)$ \\
Others & $6(3.3)$ \\
\hline
\end{tabular}

$\mathrm{AV}$, aerobic vaginitis. unemployed women in the $\mathrm{AV}$ group was higher than in the control group ( $24.7 \%$ vs. $16.7 \%, \mathrm{P}=0.039)$.

The distribution of bacterial pathogens in pregnant women with AV is summarized in Table 2. The mean gestational week at diagnosis of $\mathrm{AV}$ was $22.3 \pm 8$.6 weeks. The most common pathogen was Escherichia coli (28.6\%), followed by group B streptococcus (24.2\%), Enterococcus faecalis (17.6\%), Enterococcus faecium (8.2\%), coagulase-negative staphylococci (7.1\%), staphylococcus aureus $(6.0 \%)$, lactobacillus (4.9\%), and other pathogens (3.3\%).

Risk factors of $\mathrm{AV}$ were analyzed using logistic regression as shown in Table 3. Older age, history of cesarean delivery, history of vaginal infection, college education or above, and employment showed significant effects on the incidence of $\mathrm{AV}$ according to univariable logistic regression. However, after adjustment using multivariable logistic regression, 
Table 3 Risk factors of AV in pregnant women

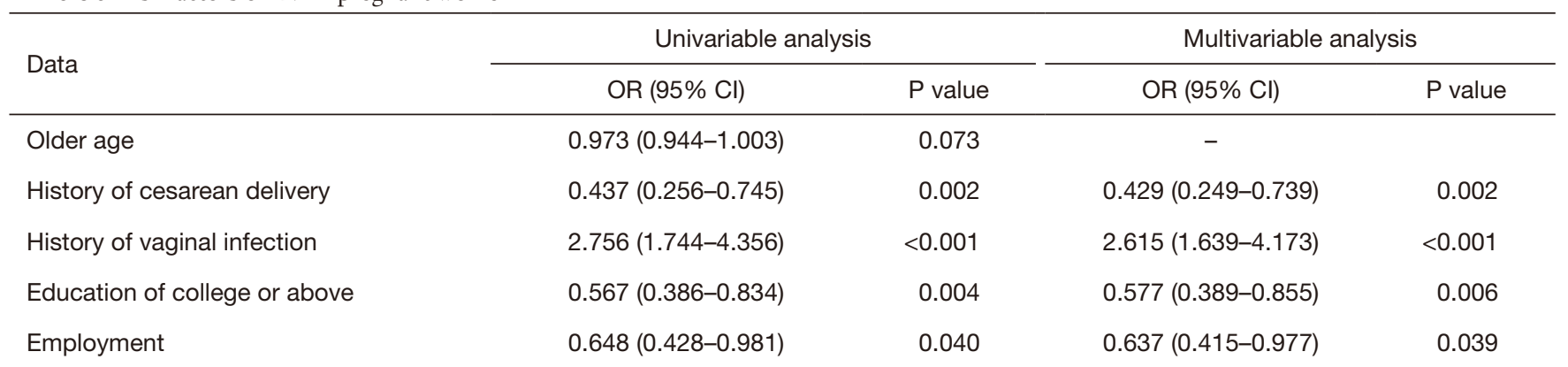

$\mathrm{AV}$, aerobic vaginitis.

Table 4 Pregnancy outcomes of women with AV

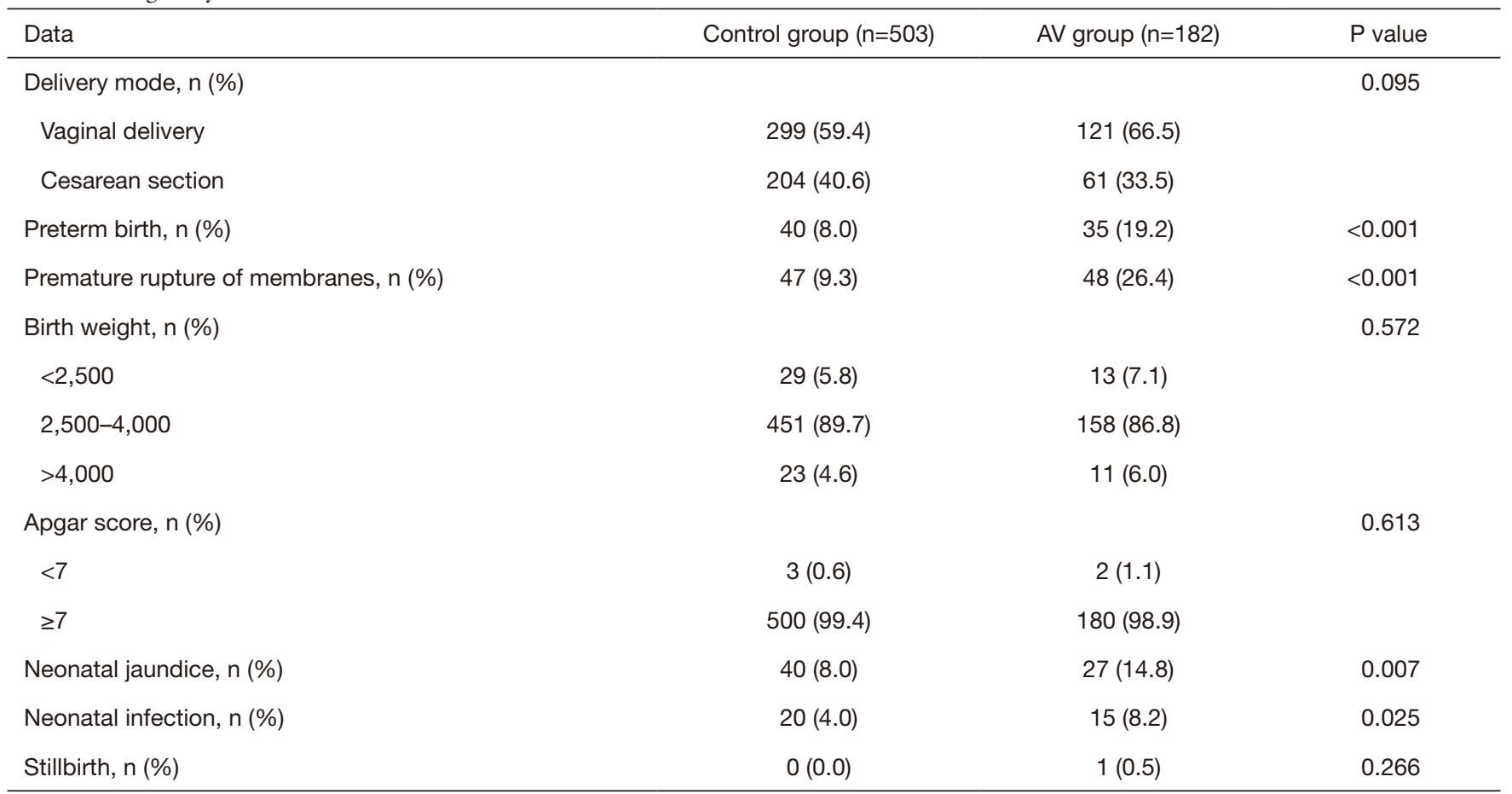

$\mathrm{AV}$, aerobic vaginitis.

a history of vaginal infection acted as an important risk factor of $\mathrm{AV}$ incidence, and a history of cesarean delivery, college education or above, and employment could protect pregnant women from the incidence of AV.

Pregnancy outcomes of pregnant women with $\mathrm{AV}$ in both groups are also listed in Table 4. Vaginal delivery took place in 299 and 121 women in the control group and $\mathrm{AV}$ group, respectively, and preterm birth occurred in 40 women $(8.0 \%)$ in the control group and 35 women $(19.2 \%)$ in the AV group, showing significant difference $(\mathrm{P}<0.001)$. Similarly, premature rupture of membranes occurred in 47 women (9.3\%) in the control group and 48 women $(26.4 \%)$ in the $\mathrm{AV}$ group, showing significant difference $(\mathrm{P}<0.001)$. Neonatal jaundice was observed in 40 neonates $(8.0 \%)$ of the control group and 27 neonates (14.8\%) of the $\mathrm{AV}$ group, showing a significant difference $(\mathrm{P}=0.007)$, and neonatal infection was observed in 20 neonates $(4.0 \%)$ of the control group and $15(8.2 \%)$ of the AV group, also showing a significant difference $(\mathrm{P}=0.025)$. Only one stillbirth was observed in all enrolled women of both groups.

Finally, the risk factors of premature rupture of 
Table 5 Risk factors of premature rupture of membranes and neonatal infection in pregnant women

\begin{tabular}{|c|c|c|c|c|}
\hline Data & \multicolumn{2}{|c|}{ Univariable analysis } & \multicolumn{2}{|c|}{ Multivariable analysis } \\
\hline \multicolumn{5}{|c|}{ Premature rupture of membranes } \\
\hline AV & $3.475(2.225-5.428)$ & $<0.001$ & 2.505 (1.537-4.082) & $<0.001$ \\
\hline Older age & $1.131(1.091-1.171)$ & $<0.001$ & 1.141 (1.099-1.184) & $<0.001$ \\
\hline Parity $\geq 1$ & $0.607(0.387-0.953)$ & 0.030 & $0.652(0.405-1.050)$ & 0.079 \\
\hline \multicolumn{5}{|l|}{ Neonatal infection } \\
\hline AV & $2.169(1.086-4.334)$ & 0.028 & $2.384(1.173-4.845)$ & 0.016 \\
\hline Older age & $1.114(1.058-1.173)$ & $<0.001$ & $1.118(1.061-1.178)$ & $<0.001$ \\
\hline
\end{tabular}

$\mathrm{AV}$, aerobic vaginitis.

membranes and neonatal infection were also analyzed and are shown in Table 5. After adjustment using multivariable logistic regression, the incidence of $\mathrm{AV}$, older age, and higher body mass index were identified as important risk factors of premature rupture of membranes. Similarly, the incidence of $\mathrm{AV}$ and older age were also important risk factors of neonatal infection.

\section{Discussion}

This retrospective cohort study enrolled 685 pregnant women from a single center. We measured the pathogen profile of $\mathrm{AV}$ in these pregnancies as well as the gestational week at diagnosis of AV. The risk factors of AV were then identified using multivariable logistic regression, and included history of vaginal infection, history of cesarean delivery, college education or above, and being unemployed. The results of this study may assist in the treatment and prevention of $\mathrm{AV}$ in pregnant women.

In recent years, Trichomonas vaginitis has been decreasing while the incidence rate of $\mathrm{AV}$ has remained stable. A total of 665 pregnant women in this study suffered from vaginitis, of which $182 \mathrm{had} \mathrm{AV}$, accounting for $27.3 \%$. The proportion of $\mathrm{AV}$ was slightly higher than that in previous studies, which ranged from $4.2 \%$ to $23.7 \%(10,12,15,16)$. Krauss-Silva et al . reported a prevalence of $32.5 \%$ of bacterial vaginitis in black women and $28.1 \%$ in white women, indicating the living environment and race could significantly affect the incidence of bacterial vaginitis (16). Furthermore, some previous studies divided $\mathrm{AV}$ patients into a slight $\mathrm{AV}$ group and severe group, which may provide more detailed analysis for its risk factors.
However, it was difficult to group participants according to the severity of $\mathrm{AV}$ in this study due to its retrospective nature. Escherichia coli was the most common pathogen of $\mathrm{AV}$ in our study, which is consistent with the results of previous studies $(10,13,17)$. This also reveals that one of the main sources of vaginitis is the gastrointestinal tract. It has been reported that both early and late pregnancy had a higher incidence rate of $\mathrm{AV}(9,10,18,19)$. The mean gestational week at diagnosis of AV in our study was $22.3 \pm 8$.6 weeks, which may show that there is no special gestational age with high risk for $\mathrm{AV}$ in pregnant women.

We then determined the risk factors of AV according to multivariable logistic regression and found the most important risk factor to be a history of vaginal infection, which increased the risk of $\mathrm{AV}$ by 2.6 times and $\mathrm{P}<0.001$. This result aligns with that of Han et al., who reported a history of vaginal infection within 1 year would increase the risk of AV by 3.2 times in pregnant women (10). Previous studies have shown intrauterine device use, external hemorrhoids, long-term antibiotic use, and frequent vaginal douching were independent risk factors for $\operatorname{AV}(10,13)$. We found higher educational level and being employed may also help reduce the incidence of $\mathrm{AV}$ in pregnant women, which is also similar to previous studies $(20,21)$. Interestingly, we found that a history of cesarean delivery may play a protective role in pregnant women with AV. This may be because vaginal delivery can lead to changes in the vaginal flora and slight structural damage, resulting in the increased risk of $\mathrm{AV}$ in future pregnancies. We found that the presence of $\mathrm{AV}$ worsened pregnancy outcomes, by increasing the incidence of preterm birth, the premature 
rupture of membranes, neonatal jaundice, and neonatal infection. A Chinese population study also confirmed that $\mathrm{AV}$ would increase the incidence of neonatal jaundice and neonatal infection (9), and a systematic review of 12 related studies showed an association between $\mathrm{AV}$ and preterm birth and premature rupture of membranes (8). However, it also found that $\mathrm{AV}$ would increase the proportion of neonates with low birth weight. Only 42 neonates with low birth weight were delivered in our study and there was no significant difference between the two groups. After adjustment using multivariable logistic regression, older age, especially older than 35 years, was also seen as an important risk factor of adverse pregnant outcomes, which is similar to the results of a previous study (22).

There are some limitations in our study. Firstly, as a retrospective study, the types of data that can be collected are relatively limited. Some other data such as the results of serological and immunological examinations could not be obtained, which may affect the final result. Secondly, follow-up and data collection were performed when women attended hospital for prenatal examination and delivery, and some risk factors of $\mathrm{AV}$ and adverse pregnancy outcomes that may have existed out of hospital and after delivery could also not be collected. Thirdly, different pathogens of $\mathrm{AV}$ were identified in our study, and it is still unclear what effect each of these pathogens has on pregnancy outcomes. Studies focusing on the effect of a single pathogen may improve the quality of future studies.

Our study enrolled 685 pregnant women including 503 healthy women and 182 with AV and found that the incidence of $\mathrm{AV}$ increased the incidence of adverse pregnancy outcomes. To reduce the incidence of $\mathrm{AV}$ in pregnancy, more attention should be paid to women with a history of vaginal infection. The results from our study provide some evidence for clinical care and treatment in pregnancy.

\section{Acknowledgments}

Funding: None.

\section{Footnote}

Reporting Checklist: The authors have completed the STROBE reporting checklist. Available at https://dx.doi. org/10.21037/apm-21-1710

Data Sharing Statement: Available at https://dx.doi. org/10.21037/apm-21-1710

Conflicts of Interest: All authors have completed the ICMJE uniform disclosure form (available at https://dx.doi. org/10.21037/apm-21-1710). The authors have no conflicts of interest to declare.

Ethical Statement: The authors are accountable for all aspects of the work in ensuring that questions related to the accuracy or integrity of any part of the work are appropriately investigated and resolved. This study was approved by the Ethics Committee of the Affiliated Wuxi Maternity and Child Health Care Hospital of Nanjing Medical University (No. 20200037) and was conducted in compliance with the ethical principles of the Declaration of Helsinki (as revised in 2013). Written informed consent was not required due to the retrospective nature of the study.

Open Access Statement: This is an Open Access article distributed in accordance with the Creative Commons Attribution-NonCommercial-NoDerivs 4.0 International License (CC BY-NC-ND 4.0), which permits the noncommercial replication and distribution of the article with the strict proviso that no changes or edits are made and the original work is properly cited (including links to both the formal publication through the relevant DOI and the license). See: https://creativecommons.org/licenses/by-nc-nd/4.0/.

\section{References}

1. Sonthalia S, Aggarwal P, Das S, et al. Aerobic vaginitis An underdiagnosed cause of vaginal discharge - Narrative review. Int J STD AIDS 2020;31:1018-27.

2. Rigo GV, Tasca T. Vaginitis: review on drug resistance. Curr Drug Targets 2020;21:1672-86.

3. Han C, Wu W, Fan A, et al. Diagnostic and therapeutic advancements for aerobic vaginitis. Arch Gynecol Obstet 2015;291:251-7.

4. Abdul-Aziz M, Mahdy MAK, Abdul-Ghani R, et al. Bacterial vaginosis, vulvovaginal candidiasis and trichomonal vaginitis among reproductive-aged women seeking primary healthcare in Sana'a city, Yemen. BMC Infect Dis 2019;19:879.

5. Tao Z, Zhang L, Zhang Q, et al. The pathogenesis of streptococcus anginosus in aerobic vaginitis. Infect Drug Resist 2019;12:3745-54.

6. Zhang HT, Wang H, Wu HS, et al. Comparison of viromes in vaginal secretion from pregnant women with 
and without vaginitis. Virol J 2021;18:11.

7. Lin Z, Lin Y, Zhang Z, et al. Systematic analysis of bacteriostatic mechanism of flavonoids using transcriptome and its therapeutic effect on vaginitis. Aging 2020;12:6292-305.

8. Juliana NCA, Suiters MJM, Al-Nasiry S, et al. The association between vaginal microbiota dysbiosis, bacterial vaginosis, and aerobic vaginitis, and adverse pregnancy outcomes of women living in Sub-Saharan Africa: a systematic review. Front Public Health 2020;8:567885.

9. Tang Y, Yu F, Hu Z, et al. Characterization of aerobic vaginitis in late pregnancy in a Chinese population: a STROBE-compliant study. Medicine (Baltimore) 2020;99:e20732.

10. Han C, Li H, Han L, et al. Aerobic vaginitis in late pregnancy and outcomes of pregnancy. Eur J Clin Microbiol Infect Dis 2019;38:233-9.

11. Novakov Mikić A, Stojic S. Study results on the use of different therapies for the treatment of vaginitis in hospitalised pregnant women. Arch Gynecol Obstet 2015;292:371-6.

12. Wang H, Huang $Z, W u Z$, et al. An epidemiological study on vaginitis in 6,150 women of reproductive age in Shanghai. New Microbiol 2017;40:113-8.

13. Geng N, Wu W, Fan A, et al. Analysis of the risk factors for aerobic vaginitis: a case-control study. Gynecol Obstet Invest 2015. [Epub ahead of print]. doi: 10.1159/000431286.

14. Pek E, Beyazit F, Korkmaz NS. Predictive value of neutrophil-to-lymphocyte ratio and platelet-tolymphocyte ratio in patients with vaginitis. Pak J Med Sci 2021;37:250-5.

Cite this article as: $\mathrm{Li}$ N, Yue Y, Chen Q. Pathogen profile and risk factors of aerobic vaginitis in pregnant women: a retrospective cohort study. Ann Palliat Med 2021;10(8):8881-8888. doi: 10.21037/apm-21-1710
15. Rumyantseva TA, Bellen G, Savochkina YA, et al. Diagnosis of aerobic vaginitis by quantitative real-time PCR. Arch Gynecol Obstet 2016;294:109-14.

16. Krauss-Silva L, Almada-Horta A, Alves MB, et al. Basic vaginal $\mathrm{pH}$, bacterial vaginosis and aerobic vaginitis: prevalence in early pregnancy and risk of spontaneous preterm delivery, a prospective study in a low socioeconomic and multiethnic South American population. BMC Pregnancy Childbirth 2014;14:107.

17. Lynch T, Peirano G, Lloyd T, et al. Molecular diagnosis of vaginitis: comparing quantitative PCR and microbiome profiling approaches to current microscopy scoring. J Clin Microbiol 2019;57:e00300-19.

18. Masukume G, Khashan AS, Kenny LC, et al. Risk factors and birth outcomes of anaemia in early pregnancy in a nulliparous cohort. PLoS One 2015;10:e0122729.

19. Du M, Liu J, Han N, et al. Maternal sleep quality during early pregnancy, risk factors and its impact on pregnancy outcomes: a prospective cohort study. Sleep Med 2021;79:11-8.

20. Schwebke JR, Gaydos CA, Nyirjesy P, et al. Diagnostic performance of a molecular test versus clinician assessment of vaginitis. J Clin Microbiol 2018;56:e00252-18.

21. Xu F, Du X, Xie L. Vaginitis in pregnancy is related to adverse perinatal outcome. Pak J Med Sci 2015;31:582-6.

22. Xie D, Xiang Y, Wang A, et al. The risk factors of adverse pregnancy outcome for pre-pregnancy couples in Hunan, China: a cross-sectional study based on population. Medicine (Baltimore) 2020;99:e23094.

(English Language Editor: B. Draper) 